\title{
Innominate Grooves of the Colon: Histological Reappraisal
}

\author{
CARLOS A. RUBIO \\ Department of Pathology, Karolinska Institute and University Hospital, Stockholm, Sweden
}

\begin{abstract}
Background: Innominate grooves (IGs) in the colon are ubiquitous structures limiting anthemic folds. The histology of the crypts in anthemic folds is well known but the histology of IGs has remained unattended. In this study, IG crypts in the normal mucosa and in ulcerative colitis (UC) were analyzed. Patients and Methods: IGs displayed a single central-lumen with merging tributary crypts. The number of merging tributary crypts, at both sides and at the bottom of the central lumen, were quantified in 60 IGs: 30 in normal colons and 30 in UC. Results: A total of 152 (median=5) tributary crypts were found merging in the 30 IGs in the normal colon, and 170 (median=5) in the 30 IGs in UC. The difference was non-significant ( $p=0.08012)$. Conclusion: Chronic inflammation did not influence the frequency of tributary crypts in IGs. The shape and the length varied considerably in the 60 IGs. Given that the chief putative physiological function of IGs is the expansion of the colon when submitted to increased intraluminal pressure, it is not inconceivable that following that demand, IGs act as hinges that permit grooves to level with the rest of the surrounding mucosa.
\end{abstract}

In 1965, William observed a fine network of lines in about $25 \%$ of routine colon barium examinations (1). The barium lines or innominate grooves (IGs), as they were named, were arranged linearly or in a reticular pattern, $0.5 \mathrm{~mm}$ apart. In 1977, Frank et al. identified marginal spiculations and punctate collections of barium in patients having a normal colon (2). The punctate pattern corresponded to the crypts of Lieberkühn which filled with barium, and the marginal spiculations to the barium retained in the IGs (2). In 1977, Matsuura et al. found innominate lines in about $90 \%$ of double-contrast examinations, when the colon was partially collapsed (3). Those lines were

This article is freely accessible online.

Correspondence to: Carlos A. Rubio, MD, Ph.D., Gastrointestinal Pathology Research Laboratory, Department of Pathology, Karolinska Institute and University Hospital, 17176, Stockholm, Sweden. Tel: +46 851774527, Fax: +46 851774524, e-mail: Carlos.Rubio@ki.se

Key Words: Colon, innominate grooves, tributary crypts, frequency, ulcerative colitis. described as superficial indentations of the mucosa with depths of $0.23 \mathrm{~mm}$. In 1978, Cole described the gross characteristics of innominate lines and grooves in the colonic mucosa with the aid of a dissecting microscope (4). Three basic mucosal patterns emerged: i) Linear circumferential, ii) network, and iii) mixed. Light microscopy showed innominate grooves running transversely across the mucosa every few millimeters, between the anthemic folds (4). Subsequently, in radiological images, Treugut et al. found that the most common localizations of IGs were the transverse colon near the hepatic flexure, and the descending and sigmoid colon (5). Rubesin et al. found a flat, featureless surface when the colonic mucosa was stretched in barium radiography (6). The colonic grooves re-appeared when the muscularis spontaneously contracted, creating a finely nodular surface. It was concluded that innominate grooves were more often seen when the colon was partially collapsed either during colonic spasm or on post-evacuation radiographs. Dissecting microphotography revealed that the grooves were clefts in the mucosa that disappeared with colonic distention. Once the colonic mucosa was stretched, a flat, featureless surface was seen. The caption in one of their illustrations reads: "The presence and visibility of the innominate grooves of the colon are a function of colonic distention" (6). Levine and Haggitt were the first to report IGs as cloverleaf-like crypts connect to a single lumen using light microscopy (7). These grooves limit the anthemic folds, built of regular crypts organized as regular 'test tubes' of similar size and shape (7).

A more recent review of the literature revealed that IGs are mentioned in many publications or described as cloverleaf-like crypts connected to a single lumen (7), giving the false impression that all IGs have a similar microscopic pattern. The possibility that IGs might vary in histological appearance in colonic mucosa with chronic inflammation has not been entertained.

Recently, we reported non-dysplastic colonic crypts in symmetric and asymmetric fission in colectomy specimens from patients with UC $(8,9)$. During those studies, we noticed that IGs adopted various architectural modalities. The question arose as to whether IGs in UC differ from those in the normal colonic mucosa.

The purpose of this survey was to study the microscopic architectural anatomy of IGs in detail, aiming to explore possible differences between normal colonic mucosa (10) and UC. 


\section{Patients and Methods}

The study material consisted of 60 consecutive IGs: 30 found in seven colectomy specimens from patients with UC (9) and 30 found in 22 normal colon segments, proximal or distal to surgically removed colonic adenocarcinoma (10).

Definitions. Histology of IGs. The microscopic anatomy of IGs was forensically analyzed. IGs were often found in a slightly depressed mucosal area with a luminal orifice connected to a single central luminal axis, to which horizontal to oblique crypts varying in length and shape usually merged at various levels. Some merging crypts were rudimentary. Merging crypts in IG were called tributaries (Figures 1-3).

Left tributary crypts: Merging crypts located on the left side of the central luminal axis.

Right tributary crypts: Merging crypts located on the right side of the central luminal axis.

Bottom tributary crypt: A tributary crypt usually found at the bottom of the central luminal axis of an IG; this tributary crypt was often lined with distinctive goblet cells. The thickness of the intervening lamina propria between the tributary crypts also varied.

Counting of tributary crypts in IGs. The number of the tributary crypts found on the left side, on the right side, and at the bottom of the central luminal axis of the IG, was separately recorded.

Statistical analysis. The two-tailed non-parametric Mann-Whitney $U$-test was applied to compare difference between IGs and normal colon mucosa. Statistical significance was defined as $p<0.05$.

\section{Results}

The shape and length of tributary crypts on both sides of the central lumen in normal segments and in UC. The shape and the length varied considerably in the 60 IGs. No apparent difference in length or shape between IGs in normal colonic mucosa and UC was found, despite the moderate to severe inflammation present in the lamina propria in UC. Examples of the shape and length asymmetry of tributary crypts found in the same side and at both sides of the central lumen in IG are shown in Figures 1 to 3.

Quantification of all tributary crypts merging in IGs in normal colonic segments and in UC. The results in Table I indicate that 152 (median=5) tributary crypts were found merging in the 30 IGs in normal colon, and 170 (median=5) in the $30 \mathrm{IG}$ in UC. The difference was non-significant $(p=0.08012)$.

Quantification of tributary crypts merging on the left side of the central luminal axis of IGs in normal colon segments and in ulcerative colitis. Table I shows that the median number of tributary crypts merging into the left side of the central luminal axis in normal colon segments was 2 and in UC was also 2. The difference between the two groups was not significant $(p=0.4009)$.
Table I. Number of tributary crypts merging into the left, right and bottom of the central luminal axis in 60 consecutive innominate grooves: 30 in normal colonic segments, proximal or distal to surgically removed colonic adenocarcinoma, and 30 in colectomy specimens with ulcerative colitis.

\begin{tabular}{llcc}
\hline Tributary crypts & Normal colon & Ulcerative colitis \\
\hline \multirow{2}{*}{ Left } & Number & 62 & 68 \\
& Mean (range) & $2.1(1-4)$ & $2.3(1-4)$ \\
\multirow{2}{*}{ Right } & Number & 63 & 75 \\
& Mean (range) & $2.1(1-4)$ & $2.5(1-4)$ \\
Bottom & Number & 27 & 27 \\
\multirow{4}{*}{ Total } & Mean (range) & $0.9(0-2)$ & $0.9(0-2)$ \\
& Number & 152 & 170 \\
& Mean (range) & $15.1(2-9)$ & $15.7(4-9)$ \\
\hline
\end{tabular}

Quantification of tributary crypts merging on the right side of the central luminal axis of IGs in normal colon segments and in $U C$. Table I also shows that the median number of tributary crypts merging into the right-side of the central luminal axis in normal colon segments was 2 , and in UC was also 2. The difference between the two groups was not significant $(p=0.13622)$.

Quantification of tributary crypts merging at the bottom of the central luminal axis of IGs in normal colon segments and in $U C$. The median number of tributary crypts merging at the bottom of the central luminal axis in normal colon segments was 1 and in UC was also 1 (Table I). The difference between the two groups was not significant $(p=0.99202)$.

\section{Discussion}

The present survey provides, for the first time, qualitative and quantitative histological data on merging tributary crypts in IG. The main findings were:

i) The histological architecture of IGs consisted of a collection of tributary crypts merging at both sides of a sagittal luminal virtual axis, and a tributary crypt merging at the bottom of the central lumen. Hence, the characteristic 'test tube' arrangement found in anthemic folds (7) was not recreated in the colonic mucosa of IG.

ii) The length and shape of the tributary crypts varied both within the same sagittal plane, between sides and at the bottom of the central lumen (cf Figures 1-3).

iii) The difference in length and shape in tributary crypts contrasted with the similar length and shape in crypts found in the anthemic folds (7) (Figure 1, left).

iv) The amount of lamina propria found between the tributary crypts varied considerably, whereas the amount between the crypts arranged as test tubes in the anthemic folds was usually similar (7). 

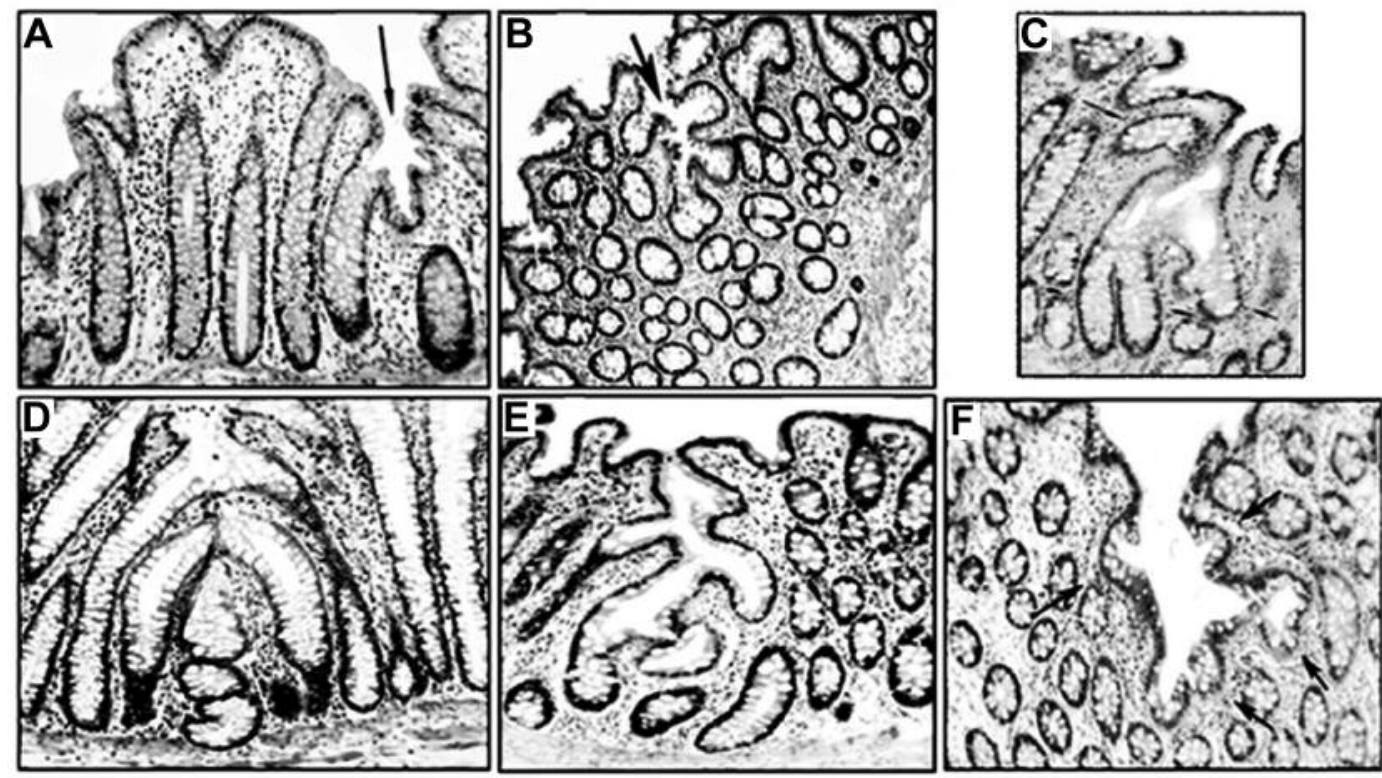

Figure 1. Innominate grooves in 'normal' colonic segments, proximal or distal to surgically removed colonic adenocarcinoma. A-F: Histological variations in number, shape and length in tributary crypts merging on both sides of the central luminal axis. Hematoxylin and eosin staining, original magnification: $A, C, D, E, F: \times 10, B: \times 4$. Examples; $A, B$ : central lumen (arrows), $C, F$ : Tributary merging crypts (arrows).
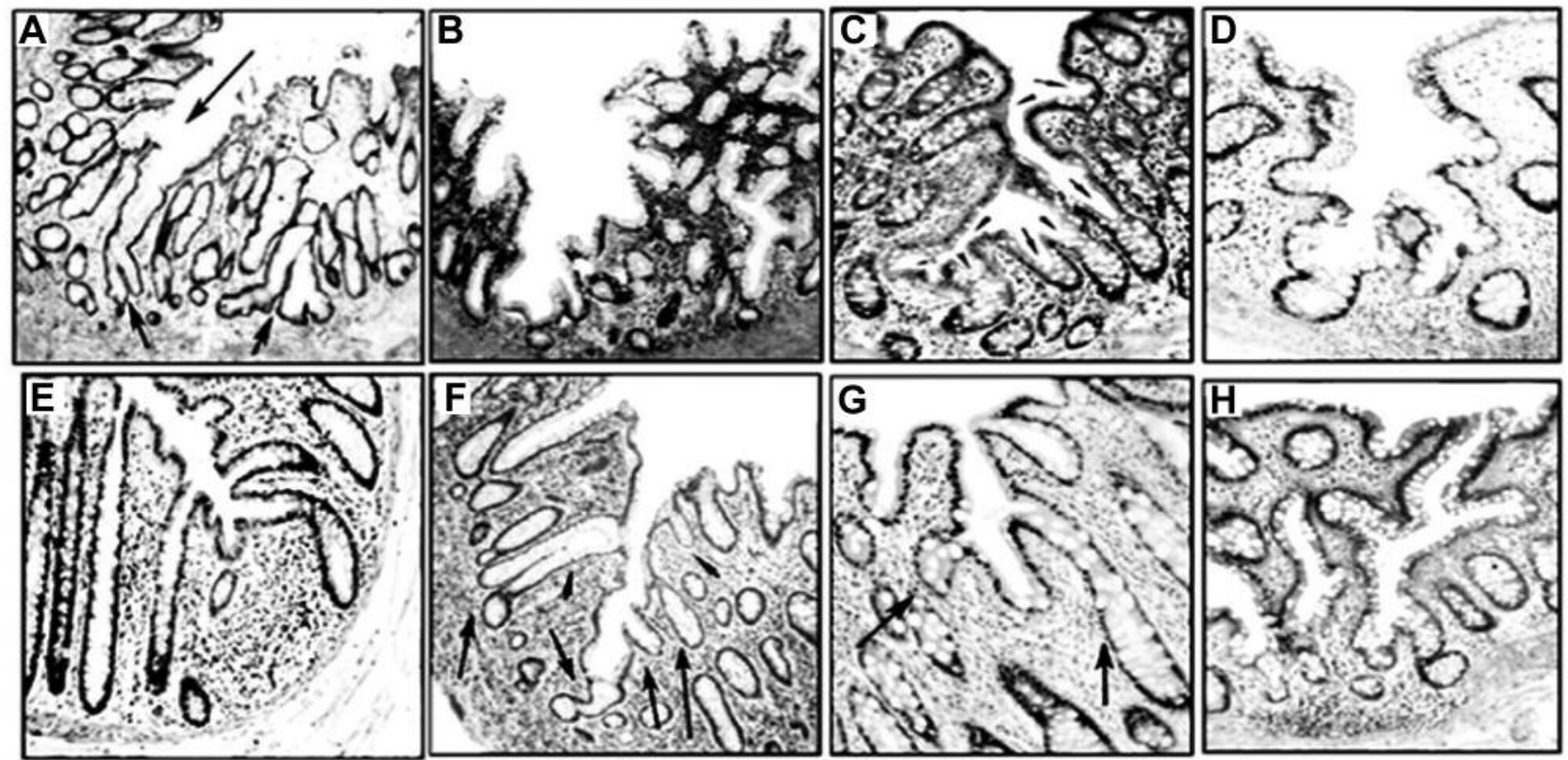

Figure 2. Innominate grooves in ulcerative colitis. A-H: Histological variations in number, shape and length in tributary crypts on both sides of the central luminal axis. Hematoxylin and eosin staining, original magnification: $A, B, C, F \times 4 ; D, E, G, H \times 10$. Examples; A: Central lumen (arrow), $C, F, G$ : Tributary merging crypts (arrows).

v) Some of the tributary crypts were 'rudimentary'. It may be argued that these rudimentary tributary crypts were not at the same cutting plane as the other tributary crypts. On the other hand, no rudimentary crypts were found in the anthemic folds. vi) Quantitative analysis revealed no particular differences in the number of tributary IG crypts found in the colonic mucosa in normal colonic segments or in UC. It would thus appear that chronic inflammation does not influence the 

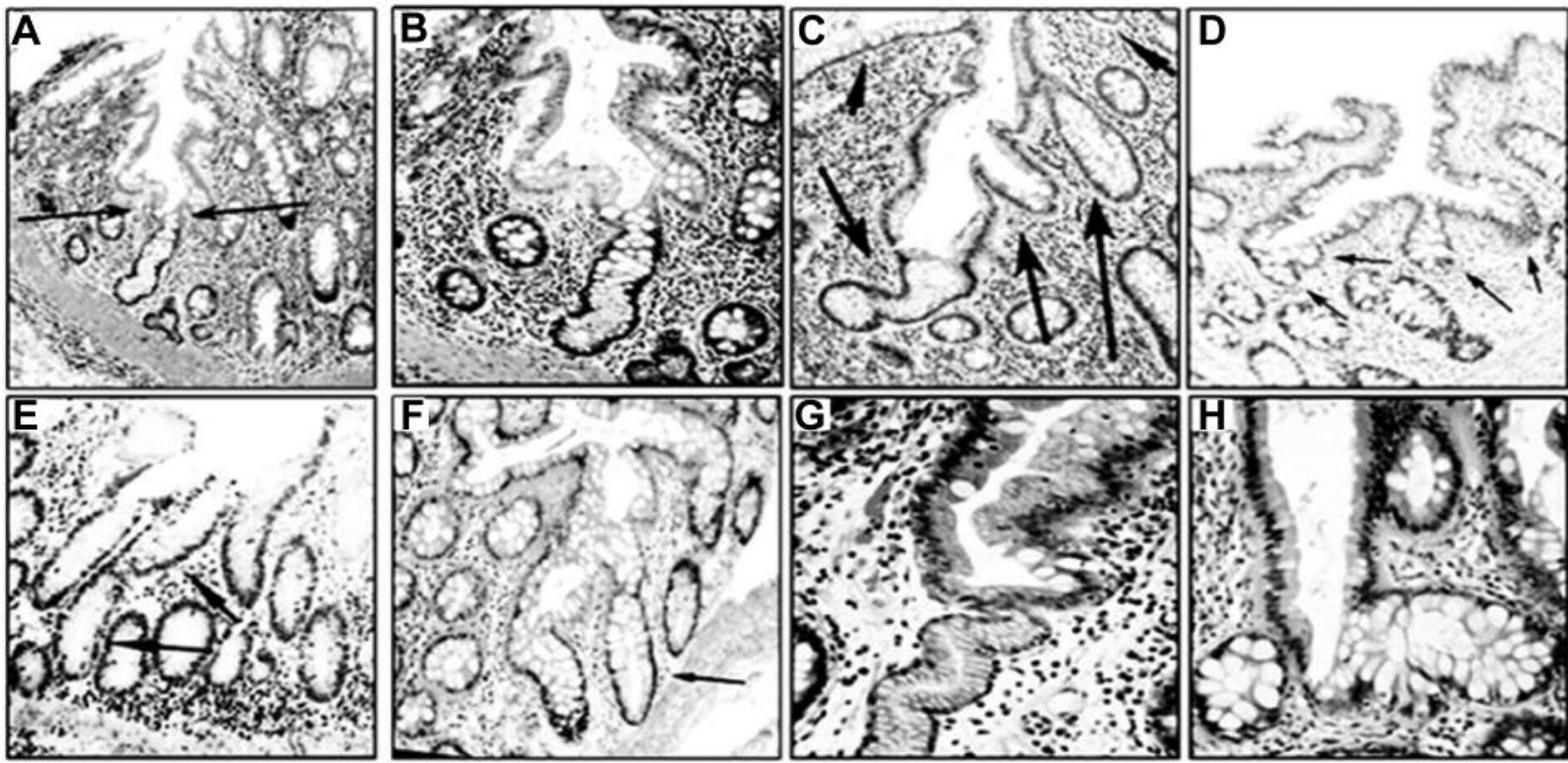

Figure 3. Hematoxylin and eosin staining of innominate grooves with tributary crypts merging at the bottom of the central luminal axis. A: Lowpower view showing distinct tributary bottom crypt merging at the arrows $(\times 4)$. B: Higher power view of the section shown in A $(\times 10)$. C-F:Other histological phenotypes of tributary bottom crypts $(\times 10) . G, H$ : Detail of tributary bottom crypts $(\times 20)$.

frequency of tributary crypts in IGs. These results are at variance with those found in anthemic folds in UC, where crypt distortions are often found (9).

The molecular signaling that orchestrates crypt replication in anthemic folds have been amply investigated; WNT is known to control cell proliferation, differentiation and apoptosis along the crypt (11); adenomatous polyposis coli (APC) forms a gradient of cellular adhesion along the crypt axis, prompting more proliferative cells to reach the top of the crypts (12); phosphatase and tensin homolog deleted on chromosome 10, a protein encoded by the phosphatase and tensin homolog (PTEN) gene, regulates crypt formation (13); and sodium-hydrogen exchanger regulatory factor 1 (NHERF1) protein controls colonic gland morphogenesis (14). On the other hand, surprisingly, no studies have been carried out to explore whether similar or different molecular signaling is required to coordinate the replication of tributary crypts of IG.

The pertinent question is do similar signaling molecules that orchestrate the required stimuli for anthemic folds also act in IG tributary crypts, or are tributary crypts in IGs programmed by a different molecular agenda? This question deserves to be answered before the true biological significance of IGs, both in UC and in the normal mucosa, can be fully understood.

The chief physiological function of IGs is the expansion of the colon when it is submitted to substantially increased intraluminal pressure (6). Accordingly, it is not totally inconceivable that following such a demand, the merging tributary crypts in IGs might act as hinges that permit the grooves to level with the rest of the surrounding mucosa

\section{Conflicts of Interest}

The Author has no conflicts of interest to declare.

\section{Author Contributions}

CAR designed the experiment, collected data, review the pathology, performed bibliographical research, compiled the photographic work and wrote the article.

\section{References}

1 William I: IGs in the surface of mucosa. Radiology 84: 877-880, 1965. PMID: 14282698. DOI: 10.1148/84.5.877

2 Frank DF, Berk RN and Goldstein HM: Pseudoulcerations of the colon on barium enema examination. Gastrointest Radiol 2: 129131, 1977. PMID: 615813. DOI: 10.1007/BF02256484

3 Matsuura K, Nakata H, Takeda N, Nakat S and Shimoda Y: Innominate lines of the colon. Radiological-histological correlation. Radiology 123: 581-584, 1977. PMID: 860026. DOI: $10.1148 / 123.3 .581$

4 Cole FM: IGs of the colon: Morphological. characteristics and etiologic mechanisms. Radiology 128: 41-43, 1978. PMID: 663222. DOI: $10.1148 / 128.1 .41$

5 Treugut H, Buck J and Zieger M: IGs. A discussion. ROFO 139: 1-8, 1983. PMID: 6409718. DOI: $10.1055 / \mathrm{s}-2008-1055830$

6 Rubesin SE, Furth EE, Rose D, Levine MS and Laufer I: The effects of distention of the colon during air-contrast barium 
enema on colonic morphology: Anatomic correlation. Am J Roentgenol 164: 1387-1389, 1995. PMID: 7754879. DOI: 10.2214/ajr.164.6.7754879

7 Levine DS and Haggitt RC: Normal histology of the colon. Am J Surg Pathol 3: 966-984, 1989. PMID: 2679155. DOI: 10.1097/00000478-198911000-00008

8 Rubio CA: Corrupted colonic crypts bordering regenerating mucosal ulcers in ulcerative colitis. In Vivo 3: 669-671, 2017. PMID: 28652436. DOI: 10.21873/invivo.11110

9 Rubio CA and Schmidt PT: Asymmetric crypt fission in colectomy specimens in patients with ulcerative colitis J Clin Pathol, 2020 (jclinpath-2020-206694, in press).

10 Rubio CA and Schmidt PT: Are non-dysplastic crypts with corrupted shapes the initial recordable histological event in the development of sporadic conventional adenomas? Anticancer Res 38: 5315-5320, 2018. PMID: 30194183. DOI: 10.21873/ anticanres. 12858

11 Boman MB and Fields JZ: An APC: WNT counter-current-like mechanism regulates cell division along the human colonic crypt axis: a mechanism that explains how APC mutations induce proliferative abnormalities that drive colon cancer development Front Oncol 3: 244-254, 2013. PMID: 24224156. DOI: $10.3389 /$ fonc. 2013.00244
12 Wasan H S, Park H S, Liu K C, Mandir N, Winnett A, Sasieni P, Bodmer W, Goolad R and Wright Nl: APC in the regulation of intestinal crypt fission. J Pathology 185: 246-255, 1998. PMID: 9771477. DOI: 10.1002/(SICI) 1096-9896(199807)185: 3<246: AID-PATH90>3.0.CO;2-8

13 Jagan IC, Deevi RK, Fatehullah A, Fatehullah A, Topley R, Eves J, Stevensen M, Loughrey M, Arthur K and Campbell F: PTEN phosphatase-independent maintenance of glandular morphology in a predictive colorectal cancer model system. Neoplasia 15: 1218-1230, 2013. PMID: 24348097. DOI: 10.1593/neo.121516 14 Georgescu MM, Cote G, Agarwal NK, White CL and Georgescu M: NHERF1/EBP50 controls morphogenesis of 3D colonic glands by stabilizing PTEN and ezrin-radixin-moesin proteins at the apical membrane. Neoplasia 16: 365-374, 2014. PMID: 24862762. DOI: 10.1016/j.neo.2014.04.004

Received November 3, 2020

Revised November 17, 2020

Accepted November 18, 2020 